\title{
Entgegnung auf die Antwort von Prof. G. Pommer und auf die Erwiderung von Prof. L. Heim.
}

\author{
Von \\ Dr. Rudolf Massini. \\ (Eingegangen am 23. März 1914.)
}

$\mathrm{Zu}$ der Antwort von Prof. Pommer habe ich folgendes $\mathrm{zu}$ bemerken: Ich möchte nur noch einmal darauf hinweisen, daß die Tabellen nur in ganz lockerem Zusammenhang mit der Arbeit stehen und daß ich zuerst beabsichtigte, überhaupt nur meine vier Bakterien, über die ich gearbeitet habe, zu bringen, wie von Hibler in seinen Tabellen nur seine 15 Arten. Nur das Fehlen einer Úbersicht in den Lehrbüchern hatte mich veranlaßt, meine Tabellen zu veröffentlichen, trotz ihrer Unvollständigkeit. Darum sind auch die 88 Arten, die nicht Gas bilden, nicht von der Liste gestrichen worden.

Das Wort ,,ungenügend" muß in dem Sinne aufgefaßt werden, wie ich es anwende. Solange noch beinahe jeder Forscher, welcher mit Anaeroben arbeitet, Verbesserungen angibt, der eine bei dieser, der andere bei jener Methode, so lange haben wir noch keine genügende Methode. Ich habe nicht behauptet, daß nicht schon Reinkulturen erhalten worden sind, sondern nur daß dies bis jetzt noch nicht leicht und sicher gelingt, daß es also einen Zweck hat, unsere alten Methoden noch zu verbessern.

Gegenüber Prof. Hei m gilt die zuletzt gemachte Äußerung ebenfalls, er selber bringt noch Verbesserungen an seiner Methode an. Des ferneren wirft er mir vor, daß ich nicht sein, sondern seine Plattenverfahren angreife. Ich muB dagegen sagen, daß ich das darum getan habe, weil die beiden Plattenverfahren in Würckers Arbeit und in seiner Arbeit (Centralblatt für Bakteriologie Bd. 55) beschrieben sind.

Da mir die Nachteile des Pyrogallolplattenverfahrens nach meinen früheren Versuchen nicht an dem Verschluß, sondern an der leichten Möglichkeit einer Verunreinigung der Kulturgefäße zu liegen schienen, habe ich wie von Hibler dieses Verfahren nicht weiter geübt, sondern ich habe mich der Botkinschen Methode zugewendet.

Nicht richtig ist ferner, daß ich die Schläuche ,entgegen der Gepflogenheit" nicht mit Paraffin überzogen habe. Allerdings habe ich 
222 R. Massini : Entgegnung auf die Antwort von Prof. G. Pommer u. L. Heim.

es unterlassen, darïber extra zu berichten, eben weil es „Gepflogenheit“" ist, gleichwie ich nicht erwähnt habe, daß zu solchen Versuchèn Vakuumschlauch benützt werden muß. Besser als Paraffin hat sich übrigens bei meinen Versuchen Hahnfett bewährt. S. 96 bemerkte ich, daß ein neuerer Kessel mit geschliffenem Deckel das Benützen von Gummischeiben unnötig macht. Oberflächenkolonien können mit dem Verfahren auch erhalten werden, nur ist das Angehen derselben nicht so absolut sicher, wie das der tiefen Kolonien.

Wenn meine Methode als unhandlich bezeichnet wird, so kann ich das zugeben dann, wenn nur wenige Platten gegossen werden. Sobald man Plattenserien eventuell zusammen mit Züchtungen in flüssigen Nährböden zu machen wünscht, so wird das Verfahren bedeutend einfacher. Wie ich schon früher bemerkte, wende ich diese Methode der Züchtung im Kessel nicht als einziges Verfahren an, sondern neben dem der hohen Schicht und neben dem der einfachen Evakuation nach Roux. Erst wenn ich mit den gewöhnlichen Methoden schwer oder unsicher zum Ziel gelange, aber gerade dann bewährt sich der Hauptvorzug des Verfahrens, die Reinlichkeit, mit der die Kulturgefäße behandelt werden. 